\title{
AC 2010-1869: A FRAMEWORK FOR USING GRAPHICAL REPRESENTATIONS AS ASSESSMENTS OF ENGINEERING THINKING
}

\section{Sean Brophy, Purdue University}

Sean $\mathrm{P}$. Brophy, $\mathrm{PhD}$. is an assistant professor of Engineering Education at Purdue University. Dr. Brophy is a learning scientist and engineer; his research focuses on the development of learners' development of adaptive expertise needed to solve complex problems in engineering, mathematics and science contexts. He uses results from his work with learners to inform instructional design methods with and without advanced technology.

\section{Sensen Li, Purdue University, West Lafayette}

Sensen Li is a graduate student in Engineering Education program at Purdue University. She received her Masters in Education Technology Program at Purdue University. Her research interest focuses on engineering problem solving, cognition and instructional design. 


\title{
A Framework for Using Graphical Representations as Assessments of Engineering Thinking
}

\begin{abstract}
Engineers and engineering students often face the challenge of comprehending complex systems because they are unsuccessful at recognizing major components in the system and the relationships between the components. Diagrams and sketches can facilitate their comprehension and communication of the complexity of a system. Their ability to construct and reason with these diagrams demonstrates their understanding of how to use the tools and their current conceptual understanding of the factors governing the behavior of that system. We are conducting a series of studies to better understand how students come to understand these tools better and its link to comprehending new domain knowledge.
\end{abstract}

This paper presents a theoretical framework for analyzing the interaction between knowledge associated with graphical representations (tools) to support thinking and the domain knowledge associated with using these tools to solve both routine problems and adapting ones knowledge to generate new knowledge (innovation). We used think aloud protocols and observation of dyads working on problem solving activities (designing, troubleshooting, analyzing or explaining) with these tools. We explored results from two pilot studies of students' generating a House of Quality and functional block diagrams, which are both useful tools in making sense of a problem context. Participants constructed the diagrams as individuals or as part of a team. Results from these studies inform the development of a framework we use to guide our interpretation of students' learning progression toward analyzing systems. In addition, the results of students' thinking will inform the design of timely and meaningful formative feedback in an automated formative assessment system called Graphical Representations to Assess System Performance (GRASP).

\section{Introduction}

Proficiency in engineering domains requires experience applying the governing principles within a specified domain and the tools needed to support the comprehension and monitoring of factors indicating a system's performance (ability to achieve a function). These tools may appear simple to describe in its form and function, but difficult to apply strategically to a context. The context is defined as strategically, because it requires a multi-step logical, systematic interaction with domain knowledge. As experts we may be blind to this interaction ${ }^{1}$; therefore, we make 
assumptions about what it takes for our students to use these tools as analytical devices to support their problem solving. Therefore, in order to make students' thinking visible ${ }^{2}$ and allow teachers to gather a variety of information about student thinking, a dynamic formative assessment system, called Graphical Representations to Assess System Performance (GRASP), is currently under development as part of a grant from the National Science Foundation. A predominate feature of this system is to engage students in manipulating graphic representation tools to facilitate their cognitive and conceptual developmental needed to analyze, describe and predict a systems' performance. For example, free-body-diagrams aid analyzing the system of forces applied to a structure, flowcharts and process diagrams illustrate signal paths through a control algorithm or transfer system (circuits, fluid systems, power distribution, feedback systems), and concept maps can represent the interdependent relationship between components in a particular system. Engineering students' thinking is made visible when they construct or manipulate these graphical representations, or graphical models, as they analyze various aspects of a system's performance. Therefore, students' abilities to construct and interpret graphical models can be used to diagnose a learner's comprehension of a system in a specific context. With this diagnostic capability we are exploring ways to provide automated formative feedback to refine students' ability to use the tool, strengthen their conceptual understanding of domain content and transform this knowledge toward the production of innovative ideas. Currently, we are in the process of conducting several pilot studies to understand how students use graphical representations to solve engineering problems. We believe the Knowledge Progression Framework presented in this paper can guide us to better interpret students' learning progression and to design timely and meaningful formative feedback tailored to their current needs.

\section{Problem statement}

One major learning objective of engineering education is acquiring knowledge to perform various actions, like design, troubleshooting and analysis ${ }^{3}$. Therefore, philosophers and educators continue to seek a useful definition of knowledge and the different ways of "knowing" this knowledge relative to how it is used in various conditions of applicability. For example, an individual can display their acquired knowledge by demonstrating knowing that something exists and knowing how ${ }^{4}$ it is used in specific context. This display of knowledge is, similar to the descriptions of declarative and procedural knowledge ${ }^{5}$. Declarative knowledge, or know that, refers to the association of objects and ideas with appropriate vocabulary that define these terms. The simplest are facts, or paired associations. This type of knowledge can be an elaborate network of facts, and can be memorization and often tested through recall tasks using multiple choices, short answer or fill in the blank. Procedural knowledge, or knowing how to perform 
specific actions, requires recall of a sequence of actions to perform an action and appropriate recall of declarative knowledge. In addition, these procedures will require the processing of declarative knowledge in order to achieve the task. A large amount of experience can lead to expert like performance of efficiently replicating and applying knowledge to perform complex actions. However, these definitions of knowing and the potential assessments used to measure this knowledge may not capture a person's ability to break from these routines and use their knowledge effectively in new situation ${ }^{6,7}$. Broudy expanded these models of knowing and proposed "knowing with", to capture the potential of flexible knowledge and reasoning abilities associated with perception, interpretation and judgment displayed in novel situation " . "Knowing with" highlights how people can generate new knowledge in situations when their prior knowledge is incomplete or when the task is so complex that an immediate solution is not self evident.

Engineering activities, and adaptive expertise, can involve all three of these types of knowledge. For example, knowing that may be learning the form and function of graphical representation tools, such as constructing a flowchart, or know-how to use the flowchart to represent components of an engineered solution. "Knowing with" the knowledge of the tool and domain knowledge related to a specific context (e.g. a complex engineering problem) can facilitate students to perceive, interpret (comprehend) and solve the problem in a correct way. The significance of how knowing with supports learning transfer in multiple implications, such as preparation for future learning, has been examined by researchers ${ }^{7}$.

Graphical representation tools for engineering activities require students to employ both graphical literacy of the tool to interpret its meaning and prior domain knowledge of a domain to appropriately link the context of the problem situation with alternative solutions. For example, most students have learned the procedure for graphing data points on a rectilinear graph as part of high school mathematics. They can also demonstrate the ability to compute the equation of line from this data. In an activity to test first year engineering students' intuition for a regression analysis we observed an interesting trend in their abilities. Students could visually interpret a best fit line that resembles what a method of least squares would define. However, when asked to identify an appropriate equation of that line, they often could not make an appropriate match. We believe this demonstrates that students may be able to perform various procedural tasks to replicate a task they have practiced (given a set of points follow a set of rules to transpose that into a graph). But when they are asked to perform a task they have not seen before, like the regression task, or to generate the equation of a line from a graph, their performance is not 
consistent. Or if they need to infer information as part of a decision relative to a domain context, then their performance using graphs is diminished.

This observation of transfer between two conditions of using two dimensional plots could be overlooked by engineering instructors, and especially when working with engineering activities supported by graphical tools. As educators we may often make assumptions of students' mastery of certain knowledge because they have had it as part of a previous service course, for example, mathematics or physics. However, one of the implications of using a constructivist theory of knowing is realizing students' knowledge is bound to a specific set of conditions used in prior experiences. This does not imply they will be able to fluently transfer this knowledge into the conditions of a new course we are teaching ${ }^{7,8}$. We need to be sensitive to the point that students are still developing their conceptual understanding and we as instructors are part of that development. Exploring these ideas in more detail requires having a comprehensive model, or framework, of knowledge associated with tools and knowledge associated with a domain.

\section{Methods for pilot studies}

Two pilot studies provided descriptive data used to inform the definition of the Knowledge Progression Framework. The studies targeted how students use graphics to troubleshoot, design and explain engineering problems. The two pilot studies involved two graphical tools: House of Quality and functional block diagrams. For both studies, participants were randomly selected from a first-year engineering course at a large mid-western university. For the first pilot study, the researcher observed team's discussion and construction of a House of Quality for the conceptual design of an aircraft. A HoQ includes multiple dimensions associated with balancing multiple criteria of a complex design task. The instructor described the basic components of the tool and explained how to complete the tool using a simple example of a backpack. This simple introduction was to orient students to the terms defining the components of the tool and how to fill in these components of the tool. Then, students worked in teams to construct their own HoQ associated with designing a game to teach aerospace design. The assumption was that students were familiar with games, and they were familiar with what needs to be learned in aerospace because they spent 8 weeks learning about the field and how to analyze sub components of an aircraft system. Teams met during a regular 50 minute class session. The students were given their own room to work in and they had access to on-line resources and a projection device to facilitate the entire teams' ability to view the HoQ diagram. The session was video recorded and researchers observed and took field notes. Initial analysis consisted of a simple coding of major transitions of students discussions as they managed the process. For example, we were looking to 
identify the sequence of steps teams took to comprehend the task and the identification of knowledge needed and synthesis of this knowledge toward to progress of constructing a diagram.

The second pilot study used a "think aloud" protocol to track students' progressive interpretation of various "systems" (e.g. automatic water level system, heat regulating incubator, automobile cruise control) using functional block diagrams. The diagrams they generated were used as secondary information to mainly understand their graphical literacy. By conducting these pilot studies, we hoped to identify when and where to provide meaningful formative feedback to facilitate students' thinking during their construction process. Additional studies on using other graphical representation tools will be investigated in the future to define a comprehensive determination of students' understanding. Initial analysis of these interviews focused on students initial moves to evaluate a system and identify how they use the representation to evaluate what will happen in various situations. Like the first pilot we are interested in identify what students know, how they use this knowledge to achieve their construction task of the diagram and how they can use the diagram to induce new knowledge (what-if conditions).

\section{Knowledge progression framework for tools and domain knowledge}

The results of these studies combined with evidence from the literature provide initial evidence for a framework we have been developing to guide our design of an assessment system. In this section we describe the Knowledge Progression Framework and the rationale for its design based on the literature on transfer, model based reasoning and our observations of students' construction and use of models related to engineering systems as displayed in the pilot studies.

These ideas of knowing that, how and with can be associated with both the knowledge of a tool (graphics in our case) and the domain knowledge associated with learning it. Also, we assume that knowledge begins with the development of declarative and procedural knowledge for both these tools and specific domain knowledge ${ }^{8}$. As expertise develops a person's knowledge develops to an efficient level where their ability to fluently use knowledge for routine procedures is demonstrated. An expert who has learned to use this knowledge adaptively will illustrate their potential for generating new ideas ${ }^{7}$. If we consider the knowledge for both must co-development, and the knowledge develops through a partially linear transition from declarative to strategic (or adaptive), then the Framework in Figure 1 could demonstrate a relationship that illustrates a pathway for how students with various knowledge skills can develop their conceptual understanding of tools and domain knowledge. 


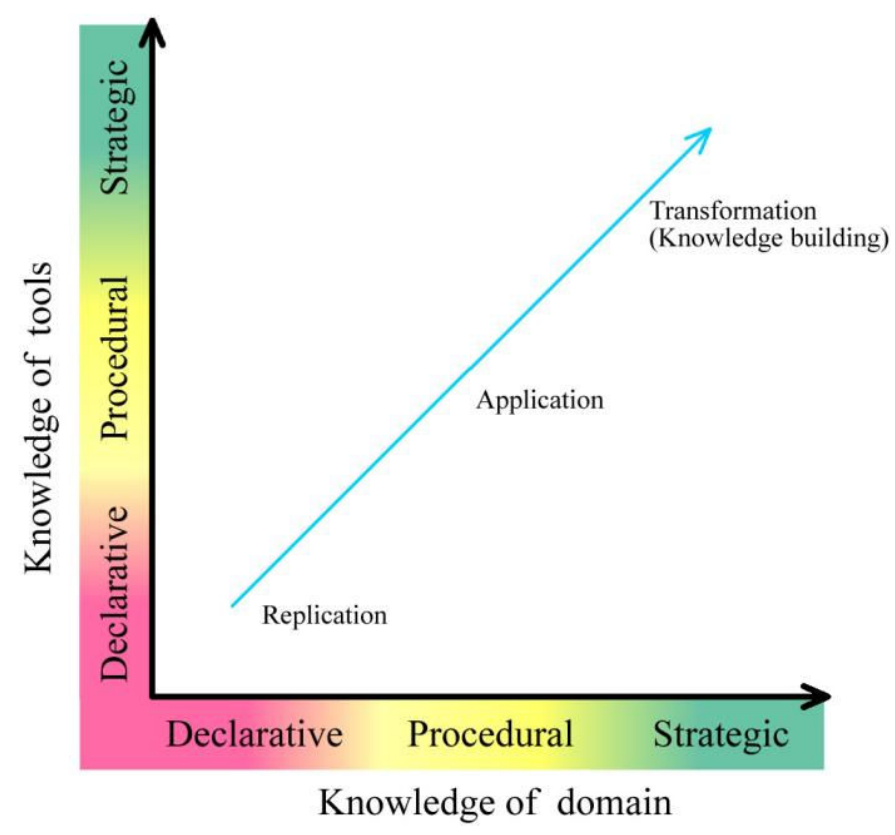

FIGURE 1- Knowledge Progression Framework to generate new knowledge with tools

Stage one: replication of declarative knowledge

When students draw graphics to solve engineering problems, many graphical representation tools illustrate the interdependence of multiple components in a complex system, and require more time and attention from students to get familiar with the tool than the domain. For example, House of Quality ${ }^{9}$ (Appendix One) is a decision making tool that aligns requirements or user needs with how these needs will be met. The graphing method also provides a mechanism to evaluate and the major interactions between these factors, and requires students to explain positive and negative impacts and the driving variables governing those decisions. This combination of all these factors can put a major cognitive load on the students. The load can be reduced by giving problems in simple or familiar domains and help them acquire more necessary declarative and procedural knowledge to understand the dimensions of the tool and how it functions. For example, how to use correct legends to symbolize the relationship between the discrete factors in a system is often neglected by engineering students.

In the first pilot study, we found early in their process many students asked questions like "What is House of Quality?", or "How do we fill in "hows'?" ("hows" being a specific set of columns in the matrix). Though these students had attended the lecture describing facts about the house of quality, they still needed scaffolding to become more acquainted with how to use the tools as 
applied to a new condition. Unfamiliarity of tools also led to the misuse of units and legends. These are important details which could be an indication that students are uncertain about both the tools and the appropriate domain knowledge needed to effectively evaluate the problem. Only a few students remembered most of the mechanics of constructing the diagram such as what are the correct use of units and legends with a HoQ. Most participants simply neglected the fact that they were working with measurable and quantifiable items. We believe these mistakes can be reduced through progressive problems sets that practicing with the tools and dealing with simple domain-related problems.

Stage two: application of procedural knowledge

When students become familiar with the graphical tools, their initial schemes are formalized by recognizing the components that the graphics represent and the potential relationship between them. Thus, students prepare to translate declarative knowledge through a process of manipulating symbols in a graphical representation. When their initial schemes interpret and assimilate a new experience, such as a contextualized engineering problem, it may either reinforce the perspective or gradually stretch its boundaries. As a result, this new experience is either rejected or transformed in order to fit into existing schemes ${ }^{10}$. Students' prior knowledge about the problem itself may either facilitate their comprehension and communication of complex systems, or lead to misconceptions of systems and eventually fail to solve the problem. For example, in one of our pilot studies, students were instructed on what are and how to use Functional Block Diagram (definition is given in Appendix Two) before they participated in the experiment. They gained the basic declarative knowledge about this graphical tool, such as the major components and features of the functional block diagram. When they participated in our experiment to use functional block diagrams to solve four engineering problems, many of them began with recalling the definition of functional block diagrams, and tried to identify and translate the "input" and "output" from the problem statement to the diagrams. Several referred to flowcharts which they have used regularly and have similar characteristics with functional block diagram. Their initial schema about functional block diagrams are primarily declarative knowledge associated with several contexts used as examples to provide definitional information. Therefore, more practice of how to use graphic tools to solve simple engineering problems may bridge the gap between knowing about the tools and how to use them in the services of problems. By practicing, students could have the opportunities to adapt their initial schemas to multiple contexts to gain a more refined knowledge of the tool that allows them to be strategic with the tool. 
Stage three: knowledge transformation for strategic knowledge

Graphical representations are used in engineering problem solving to help manage the complexity of domain knowledge (declarative and procedural) so new insights and knowledge can be achieved (knowing with). This point is prominently revealed in the strategic stage. After gaining declarative and procedural knowledge, students have to adjust their schemas again to incorporate strategic knowledge. Such strategic knowledge is often associated with complex tasks that are often open-ended. Therefore, students need to identify relevant knowledge associated with a solution to a problem and determine ways to gather knowledge they don't have but recognize the need for that knowledge, and systematically deliberate the alternatives to make a final decision on the optimized solution. Tools can facilitate this process of knowledge management and decision making. In addition the output of many tools help express the rationale for decisions which provides useful evidence when reporting to others outside the process. Like experts in other disciplines, experienced engineers routinely use these tools and domain knowledge efficiently and proficiently ${ }^{7}$. Their problem-solving schemas are wellorganized and easily accessed (recalled) in new situations ${ }^{11}$. However, engineering students may have difficulty applying graphical representations they are just learning and comprehending new domain knowledge simultaneously. In the second pilot study related to evaluating systems, some students were able to verbally describe "time" as an important factor to the system, but they did not know where to put this "invisible factor" in the diagram.

\section{Guidelines for effective feedback}

Formative feedback provides students with information based on the sequence of actions they used to perform a task within a specific situation. Formative feedback should help students modify their knowledge to increase their performance in future situations. Well designed feedback will sustain or increase their motivation and ultimately lead to achieving their desired learning goals. Black and Wiliam believe formative feedback can be the single most beneficial instructional intervention. Their literature on formative assessment ${ }^{12}$, identified two major types of formative feedback: directive and facilitative. Directive feedback provides comments telling students directly what needs to be revised. This could be simple reminders of the declarative and procedural knowledge associated with either the tool or the domain. Facilitative feedback provides relevant cues to guide the students towards a correct answer. Researchers appear to be converging toward the view that feedback is significantly more effective when it provides details about how to improve the answer, rather than when it just indicates if the student's answer is

right or wrong ${ }^{13}$. One of the challenges we are exploring is identifying the appropriate time to 
provide assistance about the tool and/or about the domain knowledge. As we discussed earlier, the learning objectives at any time in the stages may be focused on developing either tool knowledge, domain knowledge or both.

As mentioned above, when analyzing how students use graphical representations to solve engineering problems, one conjecture was their tool and domain knowledge developed simultaneously. Therefore, we need to be aware of the interplay between tool knowledge and content knowledge that students acquire during the three sub-stages, and then we can provide effectively feedback accordingly.

Feedback in stage one

Many graphical representation tools require students to comprehend the tool before they use the tool to solve problems in an unfamiliar domain. This claim is because many graphical representation tools have their own set of knowledge and skills needed to operate on the logic that is dependent on the domain knowledge. Illustrated in Figure 1, declarative knowledge provides the foundational knowledge for communicating and explaining how something works. Therefore, communicating formative feedback using text and narratives will require linguistic skills. The basic knowledge will also need to have some level of knowledge organization needed to facilitate the appropriate recall of knowledge for a given situations. However, students may spend more time and effort in comprehending tool knowledge than domain knowledge. As mentioned above, in our first pilot study with teams constructing a House of Quality, the majority of the feedback that students requested when they first came to the task was about the tool what the tool represented, its component and the relationship between these components. The next big challenge was interpreting how to transfer what they knew about the domain into the appropriate form the HoQ representation. In the HoQ pilot study, much time was spent by students figuring out the basic concept of the tool by asking the teaching assistant, looking for answers online or checking the previous lecture slides. They become cognitively overwhelmed due to high performance demands, the split attention of multiple learning goals, and thus they may benefit from supportive feedback designed to decrease the cognitive load. We believe providing feedback to illustrate what the tool is and how each section of the tool relates to each other in a familiar domain, then students can pass the first stage effectively, by progressively building connections between how the tool functions to manage domain knowledge related to the problem. Another conjecture is students become more proficient at talking about how to use the tool with the appropriate vocabulary and structural relationships, then the easier it will be for them to comprehend feedback presented to them using similar vocabulary and explanations. 
Feedback in stage two

The next challenge is transferring into acquiring procedural knowledge needed to manipulate the tool to represent domain knowledge in a form that supports comprehension of the domain situation and facilitates potential for new insight. Students' initial schemes of a domain are tested when they apply this knowledge to comprehend a new contextualized problem. They need to translate their declarative knowledge through a process of manipulating symbols in graphical representations. Therefore, in this stage of learning the procedural knowledge of the tool and domain the procedural feedback has two major characteristics for facilitating students' successful transfer into this second stage. First, procedural feedback guarantees the stability of the existed tool schemas by pointing out how the tool functions in an advanced level. This can be accomplished by providing examples of how to use the tool in multiple scenarios to allow students to see its applications. Second, procedural feedback elicits students' prior engineering knowledge by guiding students to progress towards the desired learning objectives. For example, one engineering problem from the first pilot study asked students to draw a functional block diagram to explain how an automobile cruise control works. Next we asked students to use their diagram to explain what would happen if the car went uphill or downhill. Many students were inspired by these questions, and began to re-think and revise their diagrams. Their revised diagrams and explanations were more thorough and sense-making. Therefore, we believe asking "contextualized" questions are a good way to provide procedure feedback. Furthermore, procedural feedback may be useful for correcting inappropriate task strategies, procedural errors, or misconceptions. In this stage, our conjecture is procedural feedback will lead to more productive use of "knowing with" both the tool and domain knowledge associated with engagement in engineering activities. The design of effective formative feedback will need to facilitate students noticing how the tool works to represent important properties of disciplinary knowledge that describe the behavior of a system. As students become proficient with the tool and how it works, then they will become more proficient at learning new domain content associated with that tool.

Feedback in stage three

Students' progression toward being strategic with their knowledge of tools and domain knowledge can be difficult for several reasons. In this stage learners are transitioning from replicating their performance on routine problems toward generating new knowledge to produce novel solutions. Engineering students may have difficulty noticing the problem in a novel 
context and know what questions they need to ask if they are to gain new knowledge of both the domain and the tool. If students are developing strong skills for using the tools and strategies for representing the domain, then they may have greater potential for noticing the questions they need to ask. In addition, using a tool to represent domain knowledge that is still relatively new could potentially add in gaining new insights. At this level students should be trying to become more strategic with their domain knowledge. Effective use of the tools could increase students' potential for insight toward interesting alternative to a problem. At this stage explicit diagnosis of good solutions is difficult to provide through an automated system. This is the stage were feedback is traditional provided by mentor to their apprentice. The term apprenticeship ${ }^{14}$ helps to emphasize the inherent context-dependent and situative nature of learning ${ }^{15,16}$, which aligns with the rationale of providing strategic feedback in the knowledge transformation stage. One paradigm of apprenticeship identified by Collins, Brown, \& Newman is called situated modeling $^{14}$, whereby teachers promote learning by making explicit their tacit knowledge or by modeling their strategies for students in authentic activity. Similarly, in the knowledge transformation stage, a cueing mechanism should be created to lead to more effective contentrelated information search and use of task strategies. Such cues will help students reject erroneous hypotheses and provide direction for strategizing ${ }^{17}$. For example, piecemeal strategy ${ }^{18}$ and analytic and constructive strategy ${ }^{19}$ were also proved to be helpful for engineering students to solve content-related problems. Therefore, strategic feedback will be essentially helpful for students to abstract from mechanical application of facts and ideas, and integrate the knowledge gained from the previous two stages to generate new knowledge.

\section{Implication}

Appropriate graphical representation tools can help engineers and engineering students better qualitatively comprehend the interdependence of multiple components in a system, quantitatively evaluate system performance, support logical reasoning and spatial ability ${ }^{19}$, and ultimately generate new knowledge toward defining a solution to a problem. We believe students use of "tools" can be a strong indicator of their "conceptual understanding" of facts and abstract principles and their ability to flexibly apply them to novel situations. Specifically, we are investigating how students progressively use graphical representations to solve engineering problems and how their knowledge about the domain is influenced by these graphical tools. In addition we are exploring how to provide formative feedback to students as they develop their knowledge of both the tool and the domain specific knowledge the graphical tools are representing. The knowledge progression framework of students' learning helps to differentiate the multiple components of students' knowledge development toward solving complex problems. 
This framework provides sufficient details to guide the design and timing of effective feedback which is one of the fundamental components of the GRASP system. For example, when arranging a sequence of learning activities, providing problems in familiar domain that includes less content knowledge to novel students can lead to faster and more productive use of representational tools and how they work. Further this conjecture suggests starting with tool proficiency first can lead to more robust and productive understanding of domain knowledge. With the right sequence of problem students could effectively develop proficiency in the use of tools to a strategic level and learn basics of the domain knowledge. With extended use of the tools to tackle open-ended and complex problems in the domain may lead to student becoming more strategic, or innovative, with their domain knowledge.

\section{Conclusion}

The Knowledge Progression Framework is designed to illustrate students' learning progression when using graphical representations (tools) to solve engineering problems involving domain knowledge associated with specific fields of engineering. Our intent is to use the framework to highlight the co-development of knowledge and offer pathways for supporting students' effective achievement of desired learning goals. The KP Framework also provides us with insights and an expressive power for identify when, and what kind of formative feedback is necessary as part of a learning progress. We also indicated different types of formative feedback should be provided in different stages. The three cognitive levels we recognized in the framework can help both the engineering instructor better examine how to facilitate students' learning progression relative to their learning objectives, and guide us to create the feedback mechanism in our GRASP system. By stratifying the knowledge acquisition stages, we can clearly see difficulties students may encounter and provide different types of appropriate feedback closely aligned with when students demonstrate their weakness in applying the tool and/or the domain knowledge. Our future research on students' cognition will focus on validating the framework by conducting several studies involving graphical tools relative to multiple domains. The graphical instruments we intend to use include flowcharting, house of quality, free body diagrams, functional block diagrams, schematics (specifically electrical circuits). We believe our framework can interest and enlighten researchers and engineering instructors to facilitate students in constructing diagrams and generating new knowledge. Further we are confident the framework will inform the design of effective formative assessment to support engineering education in multiple disciplines. This work may be interesting to researchers developing automated assessment systems and to instructors interested in how to provide formative feedback to promote deep understanding of engineering principles and how to construct diagrams to facilitate that reasoning. 


\section{Acknowledgements}

NSF Grant number \#0817486, Purdue University’s Discovery Learning Center, School of

Engineering Education, and the School of Aeronautics and Astronautics.

\section{Bibliography}

1. Nathan, M. J. \& Petrosino, A. (2003). Expert blind spot among preservice teachers. American Educational Research Journal, 40, 905-928

2. Bransford, J., Brophy, S. \& William, S. (2000). When computer technologies meet the learning sciences. Journal of Applied Developmental Psychology, 21, 59.

3. Brophy, S., Klein, S., Portsmore, M., \& Rogers, C. (2008). Advancing engineering education in the P-12 classrooms. Journal of Engineering Education 97 (3): 369-87.

4. Ryle, G. (1949). The Concept of Mind. Hutchinson, London, U.K.

5. Anderson, J. R. (2000). Cognitive psychology and its implications (5th ed.). New York: Worth.

6. Hatano, G. \& Inagaki, K. (1992). Desituating cognition through the construction of conceptual knowledge. In P. Light \& G. Butterworth (Eds). Context and Cognition. Ways of knowing and learning. (pp. 115-133). New York: Harvester.

7. Bransford, J. D., \& Schwartz, D. L. (1999). Rethinking Transfer: A simple proposal with multiple implications. Review of Research in Education, 24, 61-100.

8. Broudy, H.S. (1977). Types of knowledge and purpose of education. In R.C. Anderson, R.J. Spiro \& W.E. Montague (Eds.), Schooling and the acquisition of knowledge (pp.1-17). Hillsdale, NJ: Erlbaum.

9. Hauser, J. R. \& Clausing, D (1988). The House of Quality. Harvard Business Review, (3), 63-73.

10. Tsao, J., Takahashi, K., Olusesu, J. \& Jain, S. (2006). Transformative Learning. In M. Orey (Ed.), Emerging perspectives on learning, teaching, and technology. Retrieved Nov. 25, 2009, from http://projects.coe.uga.edu/epltt/

11. Chi, M.T.H., Bassok, M., Lewis, M., Reimann, P., \& Glaser, R. (1989). Self-explanations: How students study and use examples in learning to solve problems. Cognitive Science, 13, 145-182.

12. Black, P., \& Wiliam, D. (1998). Assessment and classroom learning. Assessment in Education: Principles, Policy \& Practice, 5(1), 7-74.

13. Williams, S. E. (1997). Teachers' written comments and students' responses: A socially constructed interaction. Paper presented at the annual meeting of the conference on college composition and communication, Phoenix, AZ. 
14. Collins, A., Brown, J. S., \& Newman, S. E. (1989). Cognitive apprenticeship: Teaching the craft of reading, writing, and mathematics. In L. B. Resnick (Ed.), Knowing, learning, and instruction: Essays in honor of Robert Glaser (pp. 453-494). Hillsdale, NJ: Lawrence Erlbaum Associates.

15. Anderson, J. R., Greeno, J. G., Reder, L. M., \& Simon, H. A. (2000). Perspectives on learning, thinking, and activity. Educational Researcher, 29, 11-13.

16. Greeno, J. G. (1998). The situativity of knowing, learning, and research. American Psychologist 53 (1): 5-26.

17. Earley, P. C. (1988). Computer-generated performance feedback in the magazines subscription industry. Organizational Behavior and Human Decision Processes, 41, 50-64

18. Hegarty, M., \& Steinhoff, K. (1997). Individual differences in use of diagrams as external memory in mechanical reasoning. Learning and Individual Difference, 9(1), 19-42.

19. Cooper, L. A., \& Mumaw, R. J. (1985). Spatial Aptitude. Individual differences in cognition, 67-94. Academic Press. 


\section{Appendix one - house of quality}

Designs are complex because of the number of factors to be considered and the interactions between these factors are difficult to remember. A House of Quality shown in figure A1.1 is a multifactor table that aligns customer requirements with the technical requirements of a project. The "roof" of the House of quality identifies the technical correlation between achieving various requirements. Some requirements reinforce each other resulting in a positive $(+)$ relationship. Other technical requirements may oppose each other resulting in a negative (-) relationship. For

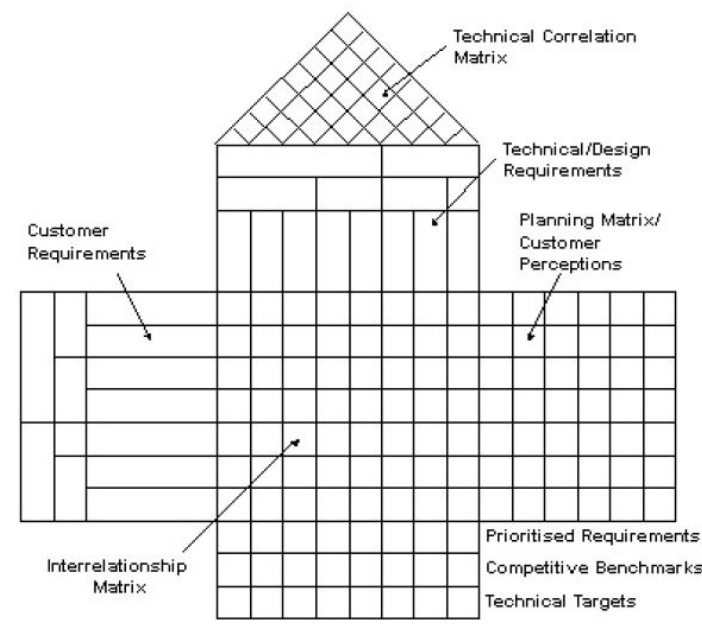

Figure A1 1 - House of quality example, increasing durability requires increase material which increases weight, but decrease weight may be an equally important requirement. These (+) and (-) relationships are placed in the roof of the house. The resulting HoQ can function as an effective executive summary of illustrating the major considerations provided in a design review. The HoQ can contain other relationships such as customers' priorities results from focus groups or surveys, and priorities on the technical requirements defined by the designers. The simultaneous interaction between all these factors can be difficult to manage without having a memory aid like the HoQ to keep track of the important factors. 


\section{Appendix two - functional block diagrams}

A Functional Block Diagram provides a visual summary of the functional decomposition of a system into its fundamental components and illustrates the relationship of these components. The diagram can show a hierarchical

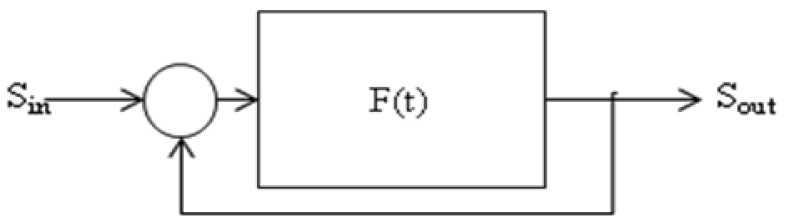

Figure A2 1 - Simple control diagram as an example of a functional block diagram relationship of responsibility and reporting such as an organizational chart used in business. In engineering these are important diagrams for outlining the basic functions of conceptual design of device or process to be constructed or implemented. The diagram can help to illustrate the basic components to be considered in a design and the interaction between these components. The diagrams can illustrate feedback mechanisms that illustrating how results from one state condition in time can change the behavior of the next state in time. A classic example in engineering is a simple transfer function shown in Figure A2.1 of a system with a negative feedback loop. The diagram illustrates how a transfer function can change (transform) its input into an output and the loop back illustrates how the relative difference between the two factors could be considered over time. 